\title{
Thermal rectification based on phonon hydrodynamics and thermomass theory
}

\author{
Yuan Dong ${ }^{\text {* }}$ \\ ${ }^{1}$ Key Laboratory for Thermal Science and Power Engineering of Ministry of \\ Education, \\ Department of Engineering Mechanics, Tsinghua University, Beijing, China \\ *Email address for correspondence: dony327@163.com \\ Communicated by Vito Antonio Cimmelli and David Jou \\ Received on November 1, 2014. Accepted on March 05, 2015.
}

\begin{abstract}
The thermal diode is the fundamental device for phononics. There are various mechanisms for thermal rectification, e.g. different temperature dependent thermal conductivity of two ends, asymmetric interfacial resistance, and nonlocal behavior of phonon transport in asymmetric structures. The phonon hydrodynamics and thermomass theory treat the heat conduction in a fluidic viewpoint. The phonon gas flowing through the media is characterized by the balance equation of momentum, like the Navier-Stokes equation for fluid mechanics. Generalized heat conduction law thereby contains the spatial acceleration (convection) term and the viscous (Laplacian) term. The viscous term predicts the size dependent thermal conductivity. Rectification appears due to the MFP supersession of phonons. The convection term also predicts rectification because of the inertia effect, like a gas passing through a nozzle or diffuser.

Keywords: thermal rectification, thermomass theory, phonon hydrodynamics, phonon Boltzmann equation

AMS subject classification: 80A20,82D37,76S05.
\end{abstract}

\section{Introduction.}

Thermal rectification, namely, the asymmetry of the thermal conductance leading to higher heat flow in one direction than in the opposite direction for the same temperature gradient, is a current active topic of research [1-11]. The thermal rectification can originate from various mechanisms, e.g. the different temperature dependences of the thermal conductivity at the different part of the device, the asymmetric transmission ratio of phonons across the interfaces, and the temperature dependence of electromagnetic resonances. Here we would like to investigate the thermal rectification related to the asymmetric geometry of the device, namely, a trapezoidal silicon flake with a wide beginning and a narrow end. The ther- 


\section{Thermal rectification}

mal rectification effect of such device has been investigated both by experiment [9] and atomistic simulation [10,11]. It is qualitatively understood that the rectification is caused by the asymmetric phonon transport caused by boundaries. Yet a more quantitative theory is desired to explain these phenomena. In recent years a series of theories are developed for the phonon transport in nanosystems. For systems evidently larger than the dominant phonon wavelength, the quantum effect can be neglected $[12,13]$. Then the phonon interaction with boundaries is characterized by the constraint of phonon mean free paths (MFPs). The equation of phonon radiative transfer (EPRT) [14] is established to solve the Boltzmann transport equation, which treats phonon like radiative photon. A predictive model (Gray model) is thus obtained for the effective thermal conductivity of nanosystems [14]. In comparing with the experimental results, the Gray model to some extent overestimates the effective thermal conductivity. It is further modified through a reconsideration of the phonon MFPs $[15,16]$, or using a frequency and branch dependent phonon group speed and mean life time [17]. Guyer and Krumahansl $[18,19]$ derived a linear solution of the phonon Boltzmann equation. They obtained a generalized heat conduction equation in the condensed matter, which contains the memory and nonlocal effect of heat flux. In particular, a Laplacian term of the heat flux appears due to the normal scattering processes of phonons. This term is in analogy with the viscous dissipation term in the Navier-Stokes equation. Therefore, models based on Guyer and Krumahansl's results are called the phonon hydrodynamic models. It was addressed that in a certain range of temperature, the collective behaviour of phonons may be characterized as in the fluid mechanics. In recent years, the significant reduction of thermal conductivity of nanosystems was exhibited in experiments [20-24]. With the phonon hydrodynamic models, the boundary drag is larger for smaller systems. Then such reduction can be explained by the boundary drag acting on the phonon gases [25-27]. The thermomass theory [28-31] derives the generalized heat conduction law from the approach of continuum mechanics. It regards the thermal energy as a continuum fluid with a certain amount of mass, which is the rest mass of energy in the relativity theory. The memory and nonlocal effects are presented as the transient and spatial inertia effects of thermomass. A phonon gas model for the decreased thermal conductivity of nanosystems is proposed by combining the phonon hydrodynamics and thermomass theory. The phonon gas is resisted by a Darcy-Brinkman friction with it viscosity depending on the systems size, which is in analogy with the rarefaction effect in micro-tubes. The previous phonon hydrodynamic and phonon gas models mainly focus on the straight tubes. In this paper, we investigate the heat transport in the trapezoidal silicon flack through these models. The 


\section{Y. Dong}

thermal rectification effects predicted by different models are presented.

\section{Thermal Rectification Based on Phonon Hydrodynamics}

The phonon hydrodynamics model is derived from the linearizd phonon Boltzamnn equation, which has a dual relaxation form [28].

$$
\left(\frac{\partial}{\partial t}+v_{k} \cdot \nabla\right) f=-\frac{f-f_{E}}{\tau_{R}}-\frac{f-f_{D}}{\tau_{N}}
$$

where $f$ is the distribution function of phonons, $\tau_{R}$ and $\tau_{N}$ are the characteristic relaxation times of resistive $(R)$ and normal $(N)$ processes, $v_{k}$ is the group velocity, $f_{E}$ is the Planck distribution for the thermal equilibrium state

$$
f_{E}=\frac{1}{\exp \left(\hbar w / k_{B} T\right)-1}
$$

$f_{D}$ is the displaced Planck distribution which conserves the phonon momentum

$$
f_{D}=\frac{1}{\exp \left[(\hbar w-\hbar \boldsymbol{k} \cdot \boldsymbol{u}) / k_{B} T\right]-1}
$$

with $u$ the drift velocity of phonon gas. Guyer and Krumalhansl $[18,19]$ obtained an eigen value solution of Eq. (1) as ( GK model)

$$
\tau_{R} \frac{\partial \boldsymbol{q}}{\partial t}+\boldsymbol{q}=-\kappa \nabla T+l_{G}^{2}\left(\nabla^{2} \boldsymbol{q}+2 \nabla \nabla \cdot \boldsymbol{q}\right)
$$

where $\kappa$ is the thermal conductivity, and $l_{G}$ is the MFP of phonons.It is worth observing that in their original proposal [18,19], Guyer and Krumhansl considered a boundary relaxation-time $\tau_{b}$ which they added to the usual relaxation time due to resistive mechanisms by the use of Matthiessen rule as $\tau_{R}^{-1}=\tau_{U}^{-1}+\tau_{i}^{-1}+\tau_{d}^{-1}+\tau_{b}^{-1}, \tau_{U}$ being the relaxation time of umklapp-phonon collisions, $\tau_{i}$ the relaxation time of phononimpurity collisions, and $\tau_{d}$ the relaxation time of phonon-defect collisions. Once the combined resistive-boundary collision time was obtained, the thermal conductivity $\kappa$ (depending on the size of the system through the $\tau_{b}$ dependence of $\tau_{R}$ ) was obtained and used in the first term of the right-hand side of Eq. 4.

Indeed, to account for the phonon-wall interactions in nanosystems, in Refs. [25-27] a different approach has been used. In particular, in those 


\section{Thermal rectification}

papers the authors assumed that the time rate of the heat flux in given by a GK-model type equation, the main differences with respect to Eq. 4 rest on the different way of computing both the resistive time $\tau_{R}$ (which does no longer account for the boundary relaxation time $\tau_{b}$ ), and the thermal conductivity $\kappa$ (chosen as the Ziman limit for the bulk thermal conductivity [32] ). Then the phonon-wall interactions are taken into account by the use of suitably boundary conditions [25-27], similar to those used in the hydrodynamics of rarefied gases.

For the steady transport in nanosystems, the first term of Eq. (4) is neglected, and Eq. (4) simplifies as

$$
\boldsymbol{q}=-\kappa \nabla T+l_{G}^{2} \nabla^{2} \boldsymbol{q}
$$

whenever the phonon MFP is smaller than the characteristic size of the system the latter term in the right-hand side of Eq. (5) can be neglected, and it reduces to the classical Fourier's law. Otherwise this expression describes a reduction of thermal conductivity because of collisions with the walls. Along with previous observations about the possible ways of accounting for the phonon-wall interactions, if in Eq. (5) the thermal conductivity is meant as the Ziman limit for the bulk thermal conductivity, then one should assume suitably boundary conditions. However, if the boundary roughness is comparable with the phonon wavelength (in which case the boundary is regarded as pure diffusive), then one may reasonably assume a non-slip boundary condition, i.e., the heat flux vanishes at the boundary. For a 2-D thin layers, the solution of Eq. (5) with the non-slip boundary is [25-27]

$$
q(y)=-\kappa \nabla T\left(1-\frac{\cosh \frac{y}{l_{G}}}{\cosh \frac{H}{2 l_{G}}}\right)
$$

where $H$ is the thickness of the layer, $y \in[-H / 2, H / 2]$ is the distance from the central line. From the integration of Eq. (6) one gets the effective conductivity of the layer [25-27]

$$
\kappa_{e f f}=\kappa\left(1-2 K n \cdot \tanh \left(\frac{1}{2 K n}\right)\right)
$$

where $K n$ is the Knudsen number, $K n=l_{G} / H$. For large systems, $K n$ is small, then the bulk conductivity is recovered. When the system size shrinks, $K n$ grows large, the effective conductivity reduces significantly from the bulk value. Note that Eqs. (6) and (7) are derived based on the non-slip boundary. In [25-27] the authors assumed the Maxwell slip boundary with the boundary slip of heat flux proportional to the gradient of heat 
flux at boundary and the MFP. The temperature dependence of the slip parameters was yielded through the experimental results. In [33] the boundary slip of heat flux was assumed to be proportional to the Fourier heat flux, $q_{0}$, and the effective MFP. The former is obtained through the Fourier's law with the same temperature gradient while the latter is a phenomenological function of Kn. Nevertheless, the boundary condition is sensitive to the practical experiment setup. Thus I only discuss the non-slip boundary condition to focus on the role of nonlinear terms on the thermal rectification. Since the cross sections of the layers studied in the experiments could be rectangles of finite width instead of infinitely wide, which is $W_{x}$, we made a modification on the $K n$ number, by defining the effective size of each cross section to be $[25-27]$

$$
R=R_{1} \cdot(\tanh \eta)^{2}, \frac{1}{R_{1}^{2}}=\frac{1}{W_{x}^{2}}+\frac{1}{H^{2}}
$$

where $K n=l / R, W_{x}$ is the width of each section, and $\eta$ is the aspect ratio, $\eta=\max \left(W_{x} / H, H / W_{x}\right)$. When $\eta \gg 1$ (about $>3$ ), the $K n$ defined in Eq. (8) is reduced to that appearing in Eq. (7). The reason for Eq. (8) is that when the aspect ratio is near 1 (i.e. for square nanowires), the phonon confinement will become stronger than in the $2 \mathrm{D}$ layer.

With Eqs. (7) and (8), we can evaluate the effective thermal conductivity of a silicon nano-flack. The investigated system size is: The thickness of the silicon layer is $100 \mathrm{~nm}(\mathrm{H})$; the widths at the narrow and the wide ends are, respectively, $60 \mathrm{~nm}(\mathrm{~N})$ and $800 \mathrm{~nm}(\mathrm{~W})$, and the separation between these ends is $3500 \mathrm{~nm}$. The difference between the temperatures imposed on both ends was of the order of $30 \mathrm{~K}$, and the average temperature (defined as the average of hot and cold end temperatures) was varied between $205 \mathrm{~K}$ and $325 \mathrm{~K}$. Note that in this case the open angle of the nano-flack is small, so the heat flux in each cross section can be regarded as quasi-1D, i.e. the $x$ component of the heat flux dominates, and the $y$ and $z$ components are negligible.Based on the quasi-1D approximation, the effective thermal conductivity as each cross section can be obtained through Eqs.(7) and (8). Note that the variation of area for each cross section leads to the evolution of $\kappa_{\text {eff }}$ along the $\mathrm{x}$ direction. The MFP increases with the temperature drop. Therefore, $\mathrm{Kn}$ in each cross section changes when the heat flux direction is reversed, leading to the rectification effect.

In Fig. 1 we show the calculated results for the effective thermal conductivity of the nano-flack at various temperatures. The MFPs of phonons are extracted from previous literatures [25-27]. It can be seen that the effective thermal conductivity greatly reduces from the bulk value $(148 \mathrm{~W}$ $m^{-1} K^{-1}$ for the room temperature silicon) because of the strong phonon 


\section{Thermal rectification}

scattering with boundaries. $\kappa_{\text {eff }}$ keeps growing with the temperature, which is an opposite trend to the bulk value. The conductivity for the WTN case (which refers to that the wide end is hotter than the narrow end) is smaller than that for the NTW case (which refers to that the narrow end is hotter than the wide end) in the temperature range above $230 \mathrm{~K}$. However, the rectification ratio is small. The maximum rectification ratio which appears at $325 \mathrm{~K}$ is about $3 \%$.

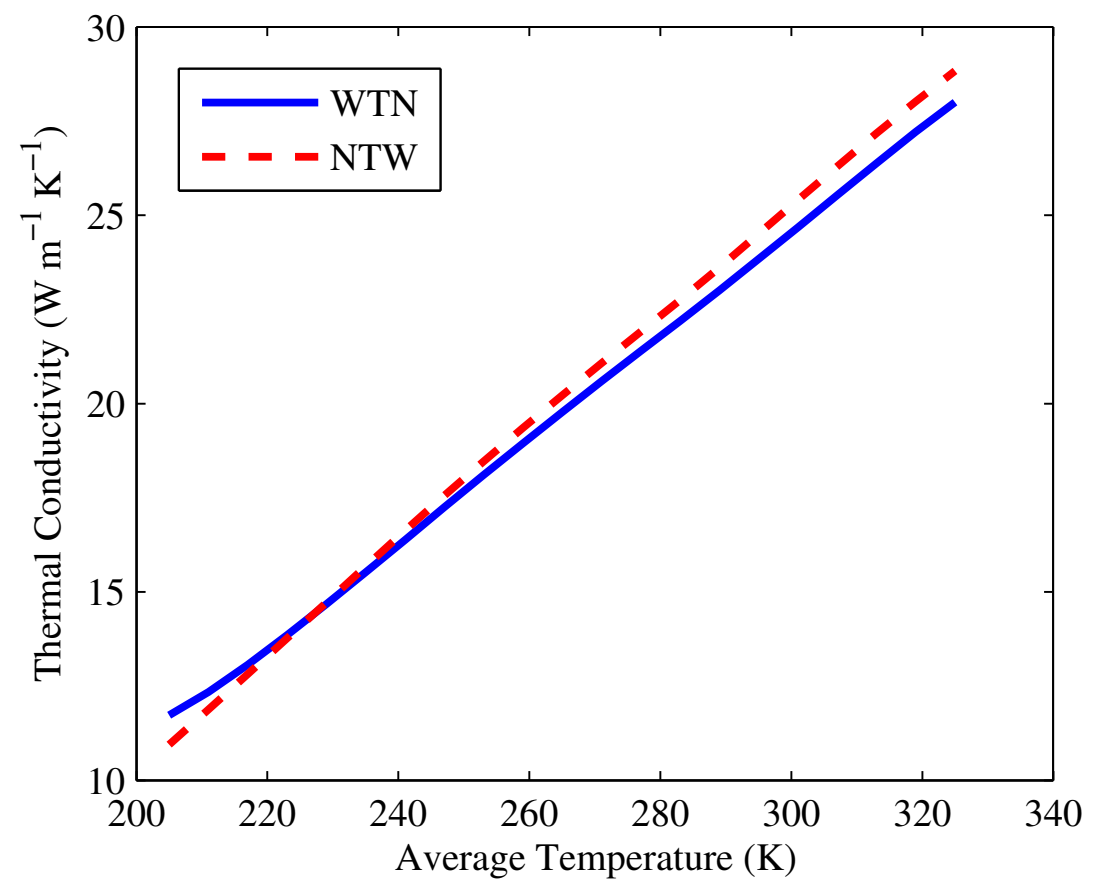

Figure 1. The effective conductivity of the trapezoidal Si flake based on the phonon hydrodynamics model (Eq. (5))

The reason for such rectification can be understood as: The phonon MFPs decrease with the temperature. In the WTN case, the narrow end contacts with the lower temperature heat bath, which has the long intrinsic phonon MFPs. Thus the constraint is stronger in this case, leading to a smaller effective thermal conductivity. This mechanism is equivalent to a device composed of two different materials which have different temperature dependent thermal conductivities. For the silicon nano-flack, the different thermal conductivities are induced by the size effect, namely, the phonon scattering with boundaries. 


\section{Y. Dong}

\section{Thermal Rectification Based on Thermomass Theory}

The thermomass theory proposes that the phonon gas residing in the condensed matter corresponds to a certain amount of mass, which is the equivalent mass of the phonon energy. The constitutive equation for the motion of thermomass is [28-31]

$$
\frac{\partial\left(\rho_{h} \boldsymbol{u}_{h}\right)}{\partial t}+\rho_{h}\left(\boldsymbol{u}_{h} \nabla\right) \cdot \boldsymbol{u}_{h}+\nabla p_{h}-\boldsymbol{f}_{h}=0
$$

which rises from the momentum balance equation of thermomass. The first term on the left hand side is the transient inertia term, the second is the spatial inertia term, the third is the driving force while the forth is the friction force. In Eq. (9) $u_{h}$ is the drift velocity of thermomass,

$$
\boldsymbol{u}_{h}=\frac{q}{\rho C_{V} T}
$$

and $\rho_{h}$ is the thermomass density

$$
\rho_{h}=\frac{\rho C_{V} T}{c^{2}}
$$

where $C_{V}$ is the specific heat and $\rho$ is the density of medium. The thermomass pressure is expressed as

$$
P_{h}=\gamma_{G} \rho_{h} C_{V} T=\frac{\gamma \rho\left(C_{V} T\right)^{2}}{c^{2}}
$$

where $\gamma_{G}$ is the Grneisen parameter. The friction force $f_{h}$ has a DarcyBrinkman form

$$
\boldsymbol{f}_{h}=-\beta \rho_{h} \boldsymbol{u}_{h}+\mu_{h} \nabla^{2} \boldsymbol{u}_{h}
$$

where $\beta$ is the friction parameter, $\mu_{h}$ is the viscosity of the phonon gas. With $\beta=2 \gamma_{G} \rho C_{V}^{2} T / \kappa$, and $\mu_{h}=2 \gamma_{G} \rho^{2} C_{V}^{3} T^{2} l_{G}^{2} / c^{2} \kappa$, Eq. (9) turns to a form

$$
\tau_{T M} \frac{\partial \boldsymbol{q}}{\partial t}+2\left(l_{T M} \cdot \nabla\right) \boldsymbol{q}-b \kappa \nabla T+\kappa \nabla T=-\boldsymbol{q}+l_{G}^{2} \nabla^{2} q
$$

with

$$
\begin{gathered}
\tau_{T M}=\frac{\kappa}{2 \gamma_{G} \rho C_{V}^{2} T} \\
\boldsymbol{l}_{T M}=\frac{\boldsymbol{q} \kappa}{2 \gamma_{G}\left(\rho C_{V} T\right)^{2}}=\boldsymbol{u}_{h} \tau_{T M}
\end{gathered}
$$




\section{Thermal rectification}

$$
b=\frac{q^{2}}{2 \gamma_{G} \rho^{2} C_{V}^{3} T^{3}}
$$

Compared with the GK model, Eq. (14) has additional terms, namely, the second and third terms on the left hand side. These terms rise from the spatial inertia term in Eq. (9). The thermomass friction force, Eq. (13), is extended with the Laplacian term compared with the previous models [28-30]. It is a necessary extension for nanosystems regarding that the friction force of porous flow should also include the Laplacian of velocity when boundary effect is important, which is called the Brinkman extension to the Darcy's law. The modified thermomass model with this extension has been used to calculate the effective thermal conductivities of the nanofilms and nanowires in [31] and got a reasonable agreement with experiments. One should note that the phonon motion could be a mixture of kinetic and collective behaviors in nanosystems. By using the hydrodynamic assumption for phonons, as in GK model and thermomass theory, the phonon is supposed to move collectively. Therefore, the parameters (e.g. $\kappa$ and $\tau$ ) used in Eq. (5) and Eq. (14) should be calculated from the collectively expression of phonon distribution function. de Tomas et al. [34] discussed the difference between thermal conductivities in the kinetic and collective regime. The general expression of thermal conductivity is a combination of both regimes with a switching factor. They obtained a better agreement with experiments. In a deliberate modeling of nanosystem heat conduction regarding the distinction between kinetic and collective regimes would give more reliable results.

In fluid mechanics it is easily found that the spatial inertia term induces the rectification effect. For a trapezoidal flow channel, the flow rate under the same pressure difference changes with the flow direction. For the WTN case, the channel serves as a nozzle, while for the NTW case it serves as a diffusor. The fluid accelerates in a nozzle, thus part of the input mechanical energy converts to the dynamical energy of the fluid, which reduces the flow rate. From this viewpoint, the NTW case will have a larger flow rate than the WTN case. However, it should be noted that in the NTW case, if the open angle is big and the flow rate is large, the flow may separate. In this case the flow rate of the NTW case will significantly decrease and the rectification effect will reverse. In steady state, Eq. (14) transforms to

$$
2\left(\boldsymbol{l}_{T M} \cdot \nabla\right) \boldsymbol{q}+\kappa \nabla T=-\boldsymbol{q}+l_{G}^{2} \nabla^{2} q
$$

Here we neglect the third term on the left hand side of Eq. (14), since it is much less than the second term in the present material and temperature 
conditions. It can be inferred from Eq. (18) that the $\nabla q$ term influences the heat conduction. In a straight channel with constant cross-sections, $q$ is constant and this term vanishes. In the WTN case, the $\nabla q$ term is positive, then it will weaken the driving force (note that $\kappa \nabla T$ is negative) and reduces the effective conductance of the device. In contrast, in the NTW case, the $\nabla q$ term is negative and enhances the effective conductance. Therefore, Eq. (18) predicts a rectification effect that the NTW case is more conductive than the WTN case.

Based on Eq. (18) we can calculate the effective conductivity of the device described in Section 2.The difference between Eq. (18) and Eq.(5) is the divergence term $2\left(\boldsymbol{l}_{T M} \cdot \nabla\right) \boldsymbol{q}$. The numerical solution of Eq. (18) is yielded by an iteration which modifies the local temperature drop and $\kappa_{\text {eff }}$ based on Eq. (5) with the divergence term. The property, $l_{T M}$, is renewed through each iteration step based on the temperature distribution obtained in the previous s tep. The relaxation time of silicon is calculated based on Eq. (15) with the temperature dependent parameters. At $300 \mathrm{~K} \tau_{T M}=1.1 e-10 \mathrm{~s}$. The results are shown in Fig. 2. The maximum rectification ratio is about $3 \%$, very close to the results based on the phonon hydrodynamics model. The reason for this consistency is that for the present device, the heat flux gradient is very small, thus the spatial inertia term is not significant to produce more rectification.

\section{Concluding Remarks}

Here we investigate the thermal rectification effect of a trapezoidal Si flack based on the phonon hydrodynamics model and thermomass model. Small rectification ratios are observed based on both models. The main reason for such rectification ratio can be attributed to the confinement of phonon MFPs. In the WTN case, the average MFPs of phonons suffer more restriction, leading to a lower thermal conductivity. However, from Figs. 1 and 2, we see that such rectification effect can reverse at different temperature ranges. At low temperature, the bulk thermal conductivity varies strongly with temperature. One can derive that putting a high conductivity material in the narrow end will benefit the total conductance. Therefore, the MFP suppression effect is no longer the main reason for rectification, and the WTN case is more conductive. The thermomass theory indicates another nonlocal term, namely, the gradient of the heat flux. Such term will cause the NTW case to be more conductive than the WTN case until the flow separation happens. In the present model such effect is not observable since the nonlocal term is not big enough. It can be expected that in the shorter systems with larger open angles, or in the more conductivity 


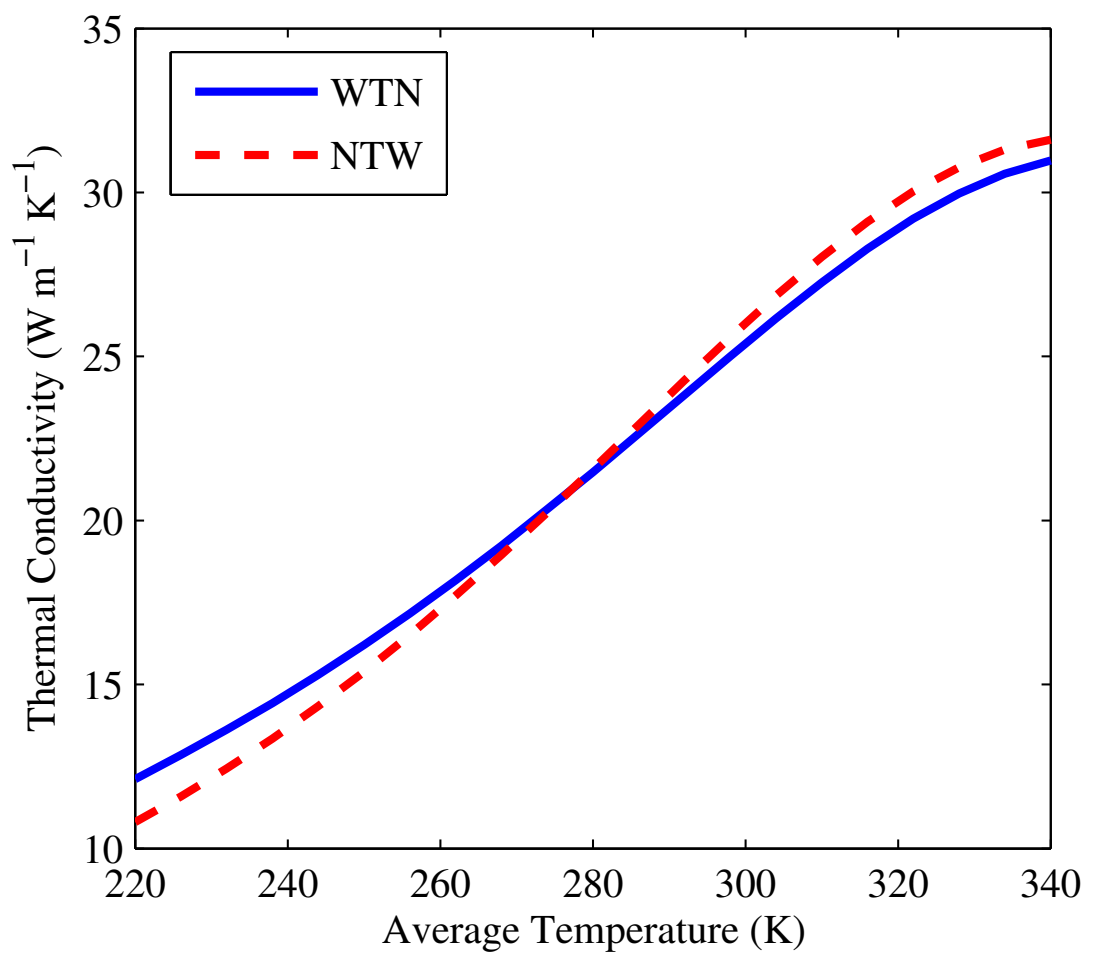

Figure 2. The effective conductivity of the trapezoidal Si flake based on the thermomass model (Eq. (18))

materials such as graphene layers, such nonlocal effect can cause considerable rectification effect. It should be noted that in the present model, we assume the nonslip boundary condition for both phonon hydrodynamics and thermomass models. It is not necessary the practical case. For the reflective boundary, the slip condition should be considered. In the trapezoidal channels, the inclined boundary may change the slip parameter due to the reflected phonons, and thereby cause a strong rectification. More investigation is underway and experimental data is desired.

\section{Acknowledgements.}

This work was financially supported by the National Natural Science Foundation of China (Nos. 51136001, 51356001) and the Tsinghua University Initiative Scientific Research Program. The calculation is supported by the Tsinghua National Laboratory for Information Science and Technology of China. 


\section{Y. Dong}

\section{REFERENCES}

1. C. W. Chang, D. Okawa, A. Majumdar, and A. Zettl, Solid-state thermal rectifier, Science, vol. 314, no. 5802, pp. 1121-1124, 2006.

2. L. Wang and B. Li, Thermal memory: a storage of phononic information, Physical Review Letters, vol. 101, no. 26, p. 267203, 2008.

3. G. Wu and B. Li, Thermal rectifiers from deformed carbon nanohorns, Journal of Physics: Condensed Matter, vol. 20, no. 17, p. 175211, 2008.

4. M. Criado-Sancho, L. del Castillo, J. Casas-Vazquez, and D. Jou, Theoretical analysis of thermal rectification in a bulk $\mathrm{Si} /$ nanoporous $\mathrm{Si}$ device, Physics Letters A, vol. 376, no. 19, pp. 1641-1644, 2012.

5. N. Yang, G. Zhang, and B. Li, Carbon nanocone: a promising thermal rectifier, Applied Physics Letters, vol. 93, no. 24, p. 243111, 2008.

6. W. Kobayashi, Y. Teraoka, and I. Terasaki, An oxide thermal rectifier, Applied Physics Letters, vol. 95, no. 17, p. 171905, 2009.

7. N. Yang, G. Zhang, and B. Li, Thermal rectification in asymmetric graphene ribbons, Applied Physics Letters, vol. 95, no. 3, p. 033107, 2009.

8. C. R. Otey, W. T. Lau, and S. Fan, Thermal rectification through vacuum, Physical Review Letters, vol. 104, no. 15, p. 154301, 2010.

9. M. Schmotz, J. Maier, E. Scheer, and P. Leiderer, A thermal diode using phonon rectification, New Journal of Physics, vol. 13, no. 11, p. 113027, 2011.

10. S. H. Ju and X. G. Liang, Thermal rectification and phonon scattering in asymmetric silicon nanoribbons, Journal of Applied Physics, vol. 112, no. 2, p. 024307, 2012.

11. S. H. Ju and X. G. Liang, Thermal rectification and phonon scattering in silicon nanofilm with cone cavity, Journal of Applied Physics, vol. 112, no. 5 , p. $054312,2012$.

12. M. Maldovan, Narrow low-frequency spectrum and heat management by thermocrystals, Physical Review Letters, vol. 110, no. 2, p. 025902, 2013.

13. R. Chen, A. I. Hochbaum, P. Murphy, and A. Majumdar, Thermal conductance of thin silicon nanowires, Physical Review Letters, vol. 101, no. 10, p. 105501, 2008.

14. A. Majumdar, Microscale heat conduction in dielectric thin films, Journal of Heat Transfer, vol. 115, no. 1, pp. 7-16, 1993.

15. G. Chen, Thermal conductivity and ballistic-phonon transport in the cross-plane direction of superlattices, Physical Review B, vol. 57, no. 23, p. 14958, 1998.

16. R. Yang and G. Chen, Thermal conductivity modeling of periodic 


\section{Thermal rectification}

two-dimensional nanocomposites, Physical Review B, vol. 69, no. 19, p. 195316, 2004.

17. A. J. H. McGaughey, E. S. Landry, D. P. Sellan, and et al., Sizedependent model for thin film and nanowire thermal conductivity, $A p$ plied Physics Letters, vol. 99, no. 13, p. 131904, 2011.

18. R. A. Guyer and J. A. Krumhansl, Solution of the linearized phonon boltzmann equation, Physical Review, vol. 148, no. 2, p. 766, 1966.

19. R. A. Guyer and J. A. Krumhansl, Thermal conductivity, second sound, and phonon hydrodynamic phenomena in nonmetallic crystals, Physical Review, vol. 148, no. 2, p. 778, 1966.

20. M. Asheghi, M. Toulzebaev, K. Goodson, Y. Leung, and S. Wong, Temperature-dependent thermal conductivity of single-crystal silicon layers in soi substrates, Journal of Heat Transfer, vol. 120, no. 1, pp. 3036, 1998.

21. Y. Ju and K. Goodson, Phonon scattering in silicon films with thickness of order 100 nm, Applied Physics Letters, vol. 74, no. 20, pp. 3005-3007, 1999.

22. W. Liu and M. Asheghi, Phonon boundary scattering in ultrathin singlecrystal silicon layers, Applied Physics Letters, vol. 84, no. 19, pp. 3819$3821,2004$.

23. Y. Ju, Phonon heat transport in silicon nanostructures, Applied Physics Letters, vol. 87, no. 15, p. 153106, 2005.

24. D. Li, Y. Wu, P. Kim, L. Shi, P. Yang, and A. Majumdar, Thermal conductivity of individual silicon nanowires, Applied Physics Letters, vol. 83, no. 14, pp. 2934-2936, 2003.

25. A. Sellitto, F. Alvarez, and D. Jou, Geometrical dependence of thermal conductivity in elliptical and rectangular nanowires, International Journal of Heat and Mass Transfer, vol. 55, no. 11, pp. 3114-3120, 2012.

26. A. Sellitto, F. Alvarez, and D. Jou, Phonon hydrodynamics and phononboundary scattering in nanosystems, Journal of Applied Physics, vol. 105, no. 1, p. 014317, 2009.

27. F. X. Alvarez, D. Jou, and A. Sellitto, Second law of thermodynamics and phonon-boundary conditions in nanowires, Journal of Applied Physics, vol. 107, no. 6, p. 064302, 2010.

28. Y. Dong, B. Y. Cao, and Z. Guo, Generalized heat conduction laws based on thermomass theory and phonon hydrodynamics, Journal of Applied Physics, vol. 110, no. 6, p. 063504, 2011.

29. M. Wang and Z. Y. Guo, Understanding of temperature and size dependences of effective thermal conductivity of nanotubes, Physics Letters $A$, vol. 374, no. 42, pp. 4312-4315, 2010.

30. M. Wang, N. Yang, and Z. Y. Guo, Non-fourier heat conductions in 


\section{Y. Dong}

nanomaterials, Journal of Applied Physics, vol. 110, no. 6, p. 064310, 2011.

31. Y. Dong, B. Y. Cao, and Z. Guo, Size dependent thermal conductivity of Si nanosystems based on phonon gas dynamics, Physica E: Lowdimensional Systems and Nanostructures, vol. 56, pp. 256-262, 2014.

32. J. M. Ziman, Electrons and phonons,. Oxford: Oxford University Press, 2001.

33. Y. Ma, Size-dependent thermal conductivity in nanosystems based on non-fourier heat transfer, Applied Physics Letters, vol. 101, no. 21, p. $211905,2012$.

34. C. de Tomas, A. Cantarero, A. F. Lopeandia, and F. X. Alvarez, From kinetic to collective behavior in thermal transport on semiconductors and semiconductor nanostructures, Journal of Applied Physics, vol. 115, no. 16, p. 164314, 2014. 